\title{
Digital Subtraction Neuroangiography: What a Resident Should Know
}

\author{
Sankar Neelakantan ${ }^{1} \quad$ Rohan Samant ${ }^{2}$ Jagadish Prasad ${ }^{1} \quad$ Bhavana Nagabhushana Reddy ${ }^{1}$ \\ Prashanth Reddy ${ }^{1}$ Bharath B. Das ${ }^{1}$ Sanjaya Viswamitra ${ }^{1}$ Dilip Mohan ${ }^{3}$
}

${ }^{1}$ Department of Radiology, Sri Sathya Sai Institute of Higher Medical Sciences, Bangalore, Karnataka, India

2Department of Radiology, UAMS, Arkansas, Texas, United States

${ }^{3}$ Department of Neruosurgery, Sri Sathya Sai Institute of Higher

Medical Sciences, Bangalore, Karnataka, India

Address for correspondence Sankar Neelakantan, MD, Department of Radiology, Sri Sathya Sai Institute of Higher Medical Sciences, EPIP area, Whitefield, Bangalore 560066, Karnataka, India (e-mail: dr.sankar@live.com).

J Clin Interv Radiol ISVIR 2019;3:44-52

\begin{abstract}
Keywords

- neuroangiography

- digital subtraction angiography

- neuroradiology

Neuroangiography (NA) is an integral investigation in the management of patients with neurovascular diseases. NA is basically used for diagnosis, but in many instances, it may be followed up with treatment in the same sitting. Indications for diagnostic NA range from extracranial diseases (vertebra-basilar insufficiency from subclavian steal, extracranial carotid stenosis, cavernous-carotid fistula, neck trauma, epistaxis, etc.) to intracranial diseases (nontraumatic subarachnoid hemorrhage, cerebral aneurysms, cerebral arteriovenous malformations, cerebral vasospasm, acute stroke, etc.). A sound understanding of the principles of appropriate periprocedural care and anatomy, catheter technique, and basic disease pathology are vital for the test to be diagnostic. This pictorial review illustrates normal neurovascular anatomy along with an approach to analyze a spectrum of neurovascular pathologies and also explains a step-wise checklist for appropriate preprocedural assessment, technique of procedure, and postprocedural care of the patient. Basic knowledge on how to interpret these images and principles on how to perform it is important.
\end{abstract}

\section{Introduction}

Neuroangiography (NA) is an integral investigation in the management of patients with neurovascular diseases. It is used for diagnosis, but in many instances, it may be followed up with interventional treatment in the same sitting. ${ }^{1}$

A sound understanding of the principles of appropriate periprocedural care and anatomy, angiography technique, and angiographic appearance of pathology are vital for the test to be diagnostic.

\section{Salient Descriptors of Normal Neurovascular Anatomy}

\section{Extracranial Carotid System (- Fig. 1)}

- Common carotid artery (CCA): Left CCA from the aortic arch and right CCA from the brachiocephalic trunk.

- External carotid artery (ECA): From the upper border of the thyroid cartilage (C4 vertebral level) to terminal branches. Branches include the following:

received

August 4, 2018

accepted after revision

September 28, 2018

published online

April 24, 2019

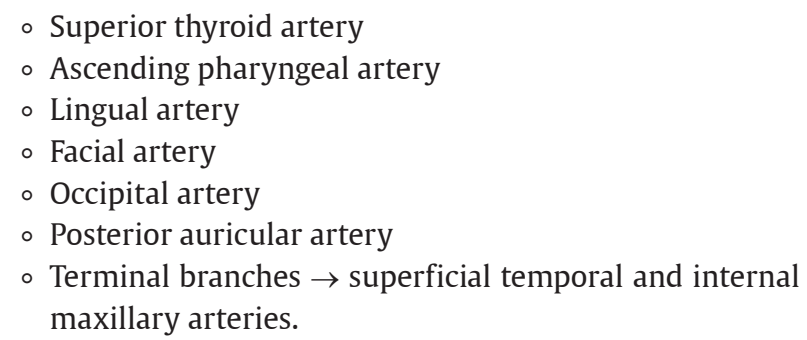

\section{Anterior Circulation (-Fig. 2)}

- Internal carotid artery (ICA)1,2: Bouthillier classification, from below upward:

- C1-Cervical segment. From the common carotid bifurcation to entry into the carotid canal.

- C2-Petrous segment. Completely within the bony carotid canal. Small branches not appreciable on digital subtraction angiography (DSA).
License terms

10.1055/s-0039-1681979

ISSN 2457-0214. 


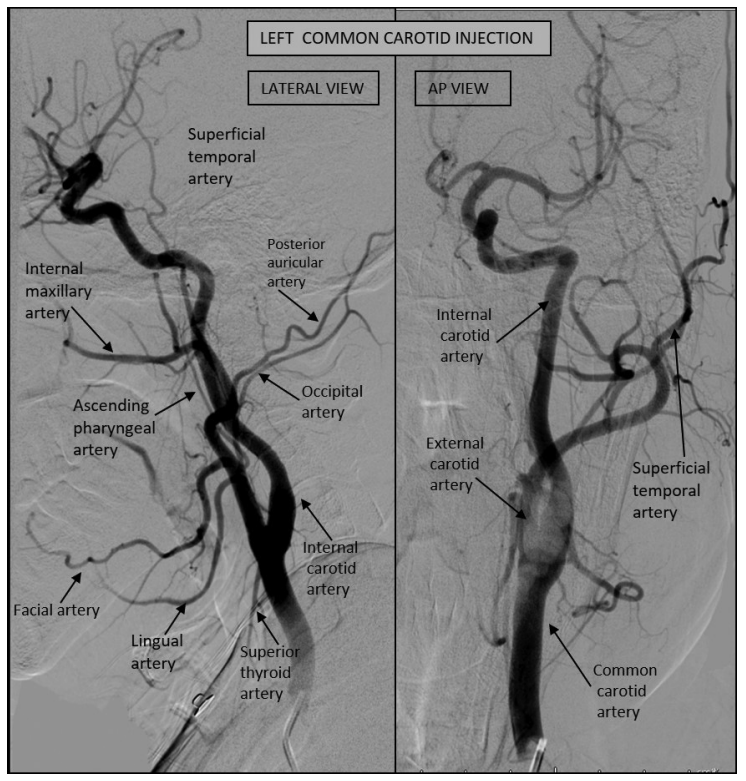

Fig. 1 Common carotid artery injection showing its primary and secondary branches.

- C3-Laceral segment. Up to the petrolingual ligament. First $1 \mathrm{~cm}$ of the ICA after exit from the carotid canal.

- C4-Cavernous segment. Up to the proximal dural rim, just inferior to the clinoid process. Branches are meningohypophyseal trunk and inferolateral trunk.

- C5-Clinoid segment. Smallest segment, by the side of the anterior clinoid segment. Ophthalmic artery can arise from it, else no significant branches.

- C6-Ophthalmic segment from distal dural ring up to the posterior communicating artery (PCOM).

- C7-Communicating from PCOM to ICA bifurcation.

\section{Physiologic Phases ${ }^{3}$}

Circulation time: time taken from contrast reaching the cavernous ICA to first cortical vein; Around 3.5 seconds is normal. It represents the physiologic perfusion time. More than 7-second period is abnormal.
Arterial, capillary (parenchymal), and venous circulation times at least in two different planes need to be assessed.

- Middle cerebral artery (MCA)

- M1-Up to insula, not the bifurcation. The bifurcation can be of the early or lateral type.

- M2-Curved course over the surface of the insula.

- M3-Descends along the circular sulcus of the insula/ operculum up to the cortical surface.

- M4-Along the convex cortical surface.

- Anterior cerebral artery (ACA)

- A1-From ICA bifurcation to anterior communicating artery (ACOM).

- A2-ACOM to origin of the pericallosal and callosomarginal artery.

- A3-Distal branches supplying the cortex.

\section{Posterior Circulation (- Fig. 3)}

- Vertebral artery

- V1-Origin from the subclavian artery to the foramen transversarium of $\mathrm{C6}$.

- V2-C6 to C2 foramen transversarium (acute bend in course).

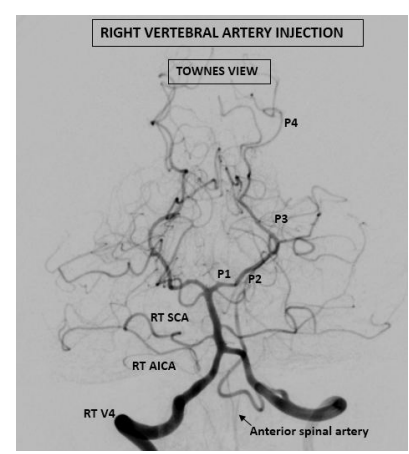

Fig. 3 Vertebral artery injection showing posterior circulation arteries. AICA, anterior inferior cerebellar artery; RT, right; SCA, superior cerebellar artery.

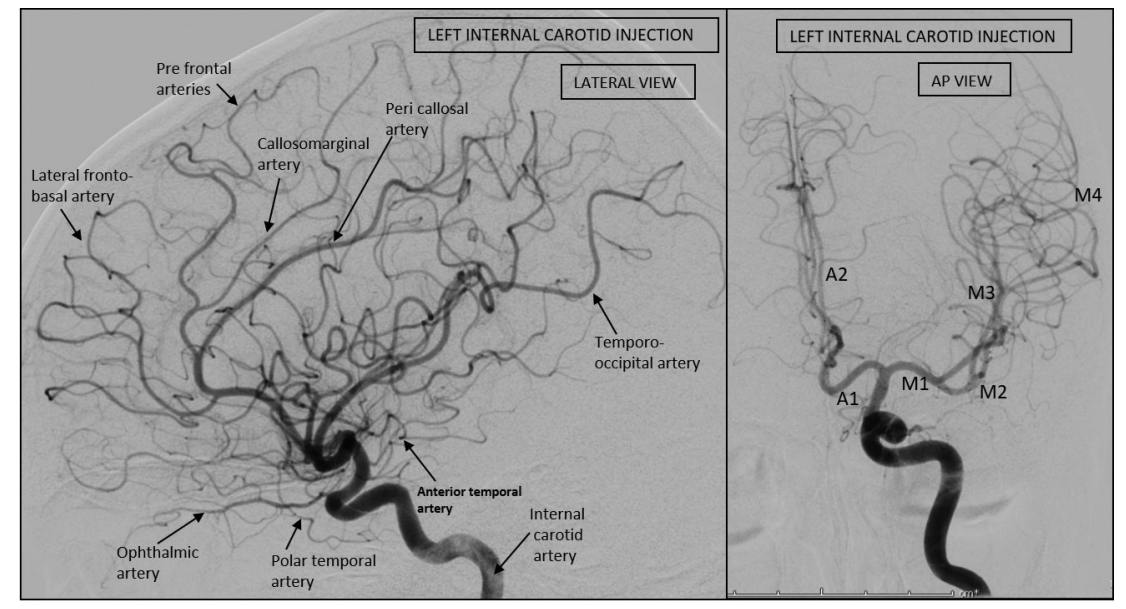

Fig. 2 Internal carotid artery injection depicting its branches. 
- V3-C2 to the dura (focal constriction), where it has a lateral, superior, and superomedial course.

- V4-Intradural part of vertebral artery, up to the formation of the basilar artery.

- Basilar artery: Branches include the following:

- Anterior inferior cerebellar artery versus posterior inferior cerebellar artery balance $\rightarrow$ supplement each other.

- Basilar perforators-not seen on DSA.

- Superior cerebellar artery

- PCOM

- P1-Basilar bifurcation to the PCOM.

- P2-Around the ambient cistern up to posterior margin of midbrain.

- P3-within quadrigeminal plate cistern.

- P4-Enters parieto-occipital and calcarine sulcus.

Branches: Thalamo perforator, posterior medial choroidal, lateral choroidal, splenial artery, and thalamogeniculate artery.

\section{Venous Anatomy}

Superficial and deep venous system are detailed and marked in the images provided (-Fig. 4).

\section{Indications ${ }^{2}$}

\section{Intracranial}

- Nontraumatic subarachnoid hemorrhage (SAH) of unknown etiology.

- Acute stroke.

- Nontraumatic parenchymal cerebral hemorrhage.

- Intracranial aneurysm: To study cross flow/complex aneurysm anatomy.

- Cerebral vasospasm.

- Mass lesions: Preoperative tumor embolization, e.g., meningioma, cavernous sinus hemangioma.

- Intracranial arteriovenous malformations to classify (Seltzer-Martin score) and plan intervention.

- Dural arteriovenous fistulas.
- Wada test.

- To obtain hemodynamic flow information-cross flow, circulation time, and collateral flow.

\section{Extracranial}

- Extracranial carotid stenosis.

- Carotid blowout.

- Subclavian steal.

- Cervical trauma.

- Epistaxis.

- Preoperative tumor embolization juvenile nasopharyngeal angiofibroma (JNA).

\section{Contraindications}

No absolute contraindications.

\section{Relative Contraindications}

- Contrast allergy

- Remedy

- Standard prophylaxis using methylprednisolone, 12 and 2 hours before the procedure.

- Low osmolar contrast media (LOCM) and judicious use of iodinated contrast.

- Pre- and postprocedure hydration with normal saline.

- Renal insufficiency: Dialysis pre- and postprocedure, if dialysis dependent.

- Coagulopathy: Should be corrected when possible.

\section{Patient Preparation ${ }^{2,4}$}

\section{Preprocedural Workup}

- Informed consent should be taken from the patient.

- Focused history with physical examination (with neurologic examination).

- Review of available imaging to assess arch anatomy or variants that may aid in catheter selection in case of vessel tortuosity/atherosclerotic disease.

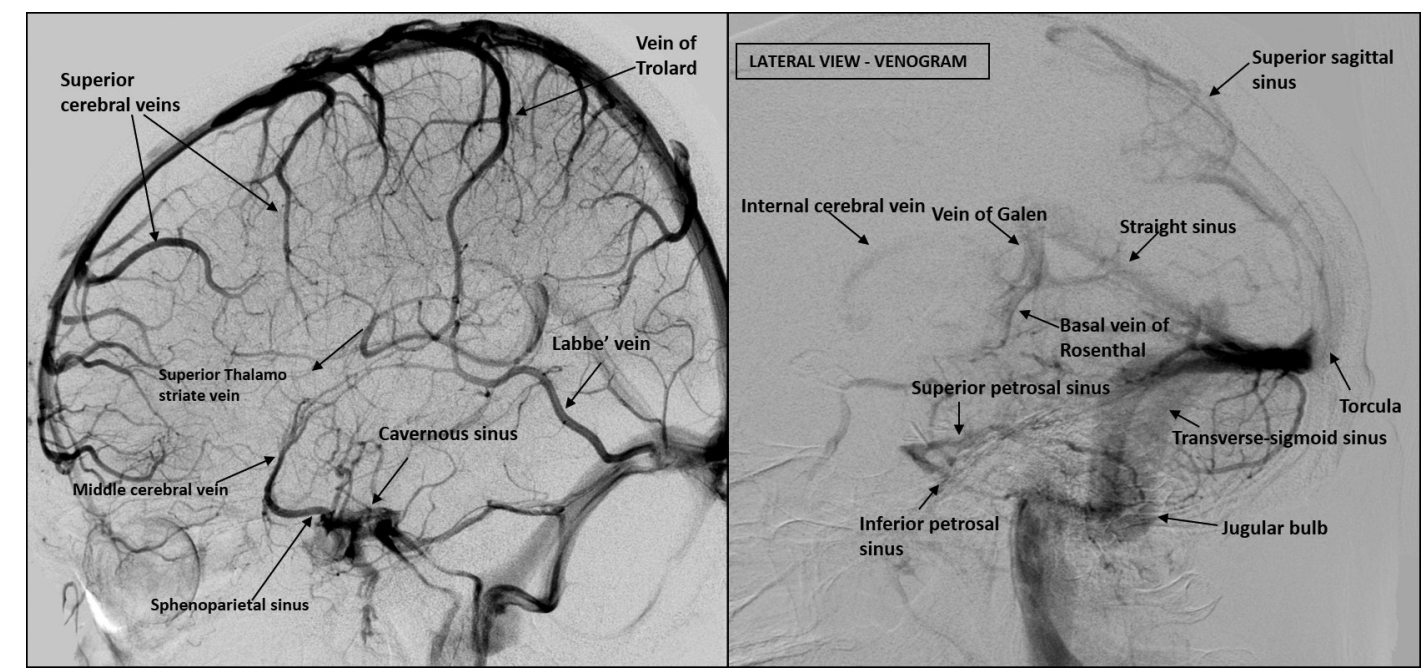

Fig. 4 Venogram depicting superficial and deep venous drainage of the brain. 
- Laboratory parameters:

- Complete blood cell count-to assess the hemoglobin status and rule out acute sepsis.

- Serum creatinine or glomerular filtration rate (GFR)for baseline record of renal status and to rule out renal dysfunction.

- Prothrombin time/international normalized ratio (PT/ INR) to rule out coagulopathy.

- Anticoagulants should be withheld when possible.

- Nil per oral for at least 6 hours preprocedure.

- The morning insulin dose should be reduced in half.

- Bilateral inguinal regions and/or the left arm (radial/ brachial access) should be prepared and made sterile depending on the case.

- An immediate presedation/anesthesia neurologic status assessment should be performed and documented.

\section{Sedation and Positioning}

- Sedation with intravenous midazolam and analgesia with fentanyl is used in our center.

- Patients should be positioned supine with a headrest, and arms are placed beside the body in extension with support.

- Uncooperative patients may need to have their head gently taped to reduce motion.

- Instructions should indicate patients to stay motionless, especially during image acquisition, and also be told about a potential sensation of warmth within the head with each injection and to avoid swallowing when imaging the neck vasculature, both aimed to reduce motion-related artifact.

\section{Technique $^{2,4}$}

\section{Access}

- Right common femoral artery (CFA) is preferred for intra-arterial access. When CFA access is not optimal, brachial artery access may be required.

- Micropuncture systems with/without ultrasound guidance versus standard 18G access needles can be used for arterial puncture, and a J-wire (atraumatic curved tip) is used, over which the arterial sheath is advanced ( - Fig. 5).

- A 5F arterial sheath is placed in situ with a continuous heparinized saline sheath infusion to prevent perisheath clotting.

\section{Catheterization}

- Catheters used

- Most often used is the $5 \mathrm{~F}$ vertebral (angled) catheter (CORDIS) ( - Fig. 5A) for normal proximal vessels.

- Judkins right coronary catheter (Terumo) ( - Fig. 5B) for old age and tortuous vessels.

- Simmons catheter (-Fig. 5C) or Mani's Head Hunter

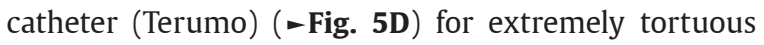
arch vessels.

- Wires: Hydrophilic 0.035-in wire such as Glidewire (Terumo).

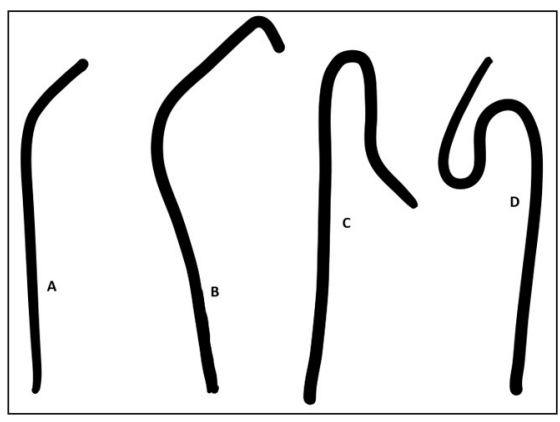

Fig. 5 Types of catheters used. (A) Angled vertebral catheter for routine cases. (B) Judkins right coronary catheter for aged patients with tortuous. (C) Simmons's catheter for extreme tortuous anatomy. (D) Mani's head hunter catheter for extreme tortuous anatomy.

\section{- Precautions}

- "Double-flush technique" $\rightarrow$ minimize unintended air or blood clot embolus.

- "Wet connect" $\rightarrow$ while connecting syringe to catheter or saline drip to sheath. This is to also prevent inadvertent air embolus.

- Tactile feedback with smooth motion on advancing wire is essential to prevent dissection/vessel perforation.

- Catheter should be always advanced over the wire, which forms support system. Torquing motion of the catheter on wire during advancing is useful.

- Road maps of the vascular anatomy should be obtained prior to crossing any vessel bifurcation, especially in cases with difficult anatomy. This aids in preventing ostial dissections.

- After advancing the catheter, as the wire is removed, an inadvertent vacuum effect if the catheter tip is wedged against the vessel wall, thus sucking in air into the catheter. To prevent air embolization, remove the guidewire slowly, keeping the catheter hub filled with heparinized saline by dripping it into the hub while the wire is withdrawn. This also aids in wet connect with the syringe after the wire is completely withdrawn.

- Prior to injection, there should be flow back from the catheter, to rule out catheter tip wedging/dissection/ intracatheter clotting.

- Extra care should be taken when selective angiogram of the vertebral artery is done, as it is prone to complications like dissection to vasospasm. Delayed or incomplete contrast washout is indicated possibility of vasospasm or dissection.

\section{Angiographic Views ${ }^{2,4}$}

- Cervical arch angiogram: especially if there is suspicion of diseased arch vessel origins (e.g., atherosclerotic narrowing of the ostium) or variant/difficult to catheterize anatomy (e.g., bovine arch).

The catheter of choice is a multiside-holed flush/pigtail catheter. The injection rate is typically 20 to $25 \mathrm{~mL} / \mathrm{s}$, and the duration of injection is 10 seconds for a total of 40 to $50 \mathrm{~mL}$ of contrast imaged at four to six frames per second (fps). Cervical aortic arch is performed approximately 
35-degree left anterior oblique position to assess the great vessel origins in profile.

- For extracranial carotid arteriography, anteroposterior (AP), lateral, and 45-degree bilateral oblique projections are standard. The injection rate is 3 to $4 \mathrm{~mL} / \mathrm{s}$ for a total of 7 to $9 \mathrm{~mL}$ of contrast imaged at two to three fps.

- For anterior intracranial cerebral angiography, AP (Towne's view) and lateral projections are standard. One helpful tip is to position the petrous bones at the level of the mid to lower orbits as a guide. The injection rate is 6 to $7 \mathrm{~mL} / \mathrm{s}$ for $10 \mathrm{~mL}$ total contrast, imaged at two to four fps.

- For vertebral arteriograms, the standard projections are AP (Towne's view) and lateral projections centered caudally and dorsally to cover the posterior circulation. In distinction to the anterior circulation, the petrous bones should be projected at the bottom or below the orbits to best visualize the basilar artery and its branches in the AP dimension. The injection rate is 3 to $5 \mathrm{~mL} / \mathrm{s}$ for total of $8 \mathrm{~mL}$ total, imaged at two to four fps (-Table $\mathbf{1}$ ).

\section{Postprocedural Care ${ }^{2,3,5}$}

- Common femoral artery access care: Hemostasis may be accomplished with manual compression or a

Table 1 Anatomic structures with specific angiographic views

\begin{tabular}{|c|c|}
\hline Anatomic structure & Specific angiographic view \\
\hline Carotid bifurcation & Posteroanterior (PA), oblique \\
\hline $\begin{array}{l}\text { Internal carotid } \\
\text { artery (ICA) cavern- } \\
\text { ous and ophthalmic } \\
\text { segments }\end{array}$ & Caldwell, lateral \\
\hline Rest of the ICA & $\begin{array}{l}\text { Anteroposterior (AP) (0 degree) } \\
\rightarrow \text { superior orbital margin and the } \\
\text { petrous bone will overlap and proj- } \\
\text { ect the supraclinoid ICA, MCA, and } \\
\text { anterior cerebral artery (ACA) above } \\
\text { the bone margin } \\
\text { Lateral } \\
\text { Oblique ( } 25-35 \text { degrees) } \rightarrow \text { To } \\
\text { assess ACA, ACOM, and MCA } \\
\text { bifurcation }\end{array}$ \\
\hline $\begin{array}{l}\text { Posterior communi- } \\
\text { cating artery }\end{array}$ & Lateral \\
\hline $\begin{array}{l}\text { Anterior commu- } \\
\text { nicating artery } \\
\text { (ACOM) }\end{array}$ & $\begin{array}{l}\text { Submentovertical } \rightarrow \text { projects ACOM } \\
\text { above the nasal cavity } \\
\text { Oblique ( } 25-35 \text { degrees) }\end{array}$ \\
\hline $\begin{array}{l}\text { Middle cerebral } \\
\text { artery }\end{array}$ & Transorbital corresponding oblique \\
\hline $\begin{array}{l}\text { Vertebral artery and } \\
\text { posterior cerebral } \\
\text { artery (PCA) }\end{array}$ & $\begin{array}{l}\text { AP ( } 20 \text { degrees caudal) } \rightarrow \text { petrous } \\
\text { bone overlaps the inferior margin } \\
\text { of the orbit, so distal vertebral and } \\
\text { basilar arteries will be in profile } \\
\text { Towne's; AP for PCA to be seen in } \\
\text { profile } \\
\text { Lateral }\end{array}$ \\
\hline Basilar artery & AP (20 degrees caudal), lateral \\
\hline
\end{tabular}

percutaneous closure device. Groin hematoma should be looked for during intensive care unit (ICU) monitoring. Puncture site vessels should be immobilized for at least 24 hours post puncture.

- Postprocedural neurologic examination should be performed and any new neurologic deficits should be documented.

- Significant neurologic changes may require further evaluation with magnetic resonance imaging (MRI) or repeat DSA to rule out acute stroke/vessel dissection, etc.

- Oral analgesics (paracetamol) could be administered in case of puncture site pain.

\section{Complications and Remedies}

- Most common complication is groin hematoma; seen in around $4 \%$ cases.

- Neurologic complications within 24 hours (transient ischemic attack) in $2.5 \%$ cases.

- Stroke with permanent focal neurologic defect in $0.1 \%$ and death in $0.06 \%$.

\section{Predictors of Complications}

- Patient related

- Subarachnoid hemorrhage evaluation

- Atherosclerotic cerebrovascular disease

- Frequent transient ischemic attacks

- Age > 55 years

- Diabetes, especially if poorly controlled

- Procedure related

- Increase in length of the procedure

- Increased number of catheter exchanges

- Larger size of catheters

\section{Reporting Essentials}

- At least two views: AP and lateral ( \pm oblique/specific views).

- Two systems: anterior and posterior circulations.

- Three phases: arterial, capillary (or parenchymal), and venous phases.

- Variant anatomy to be addressed.

- Collaterals $\rightarrow$ Primary (from ACOM and PCOM) and secondary (pial-pial and leptomeningeal-dural); compression studies to assess collateral status.

- Shifting, distortion, or herniation of the vessels.

- Lesion $\rightarrow$ tumor or vascular: description, type, etc.

\section{Case Examples 5}

We have included various common case examples (-Fig. 6-20) where DSA is useful in the diagnostic evaluation, followed by endovascular management. 


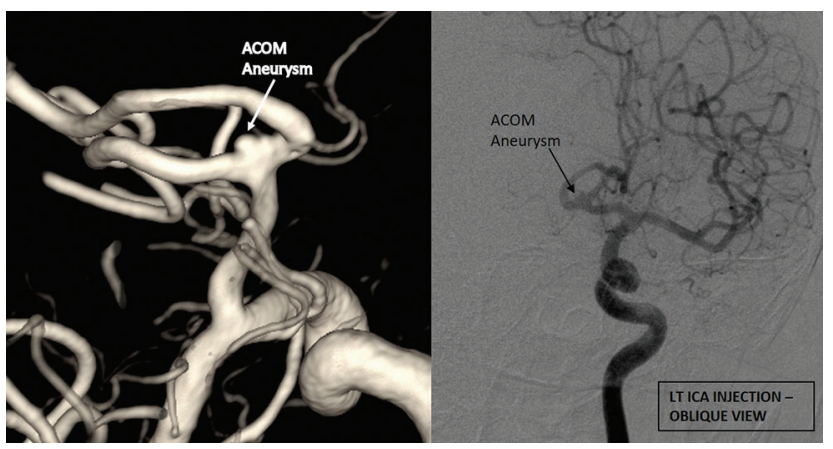

Fig. 6 There is evidence of a blister-like saccular anterior communicating artery (ACOM) aneurysm (arrow) on left internal carotid artery (ICA) injection, directed superiorly. The A2 segment of the right ACA is filling through the ACOM. Partial thrombosis of the aneurysm is also noted.

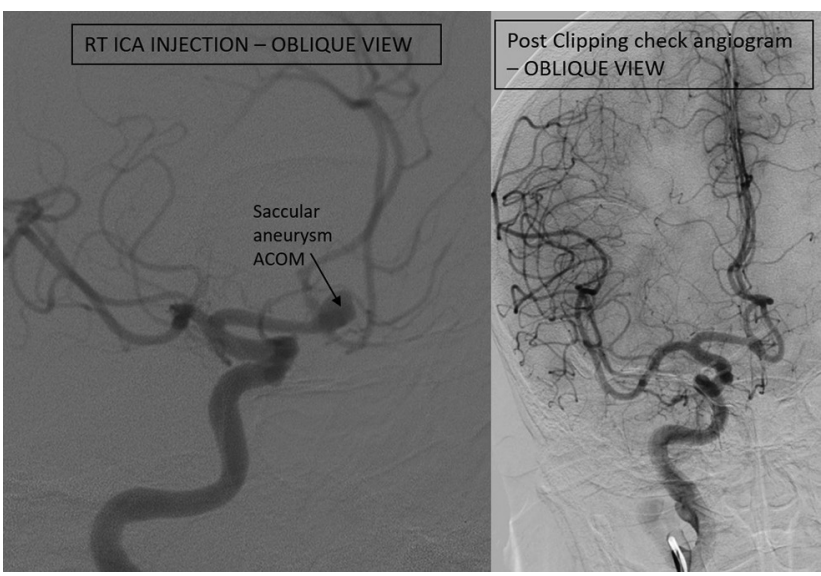

Fig. 7 Saccular anterior communicating artery (ACOM) aneurysm (arrow) noted with narrow neck. Distal ACA filling is within normal limits. Post clipping check angiogram showing no residual aneurysm sac or vasospasm. Normal parent vessels. Faint clip artifact is also present. ICA, internal carotid artery.

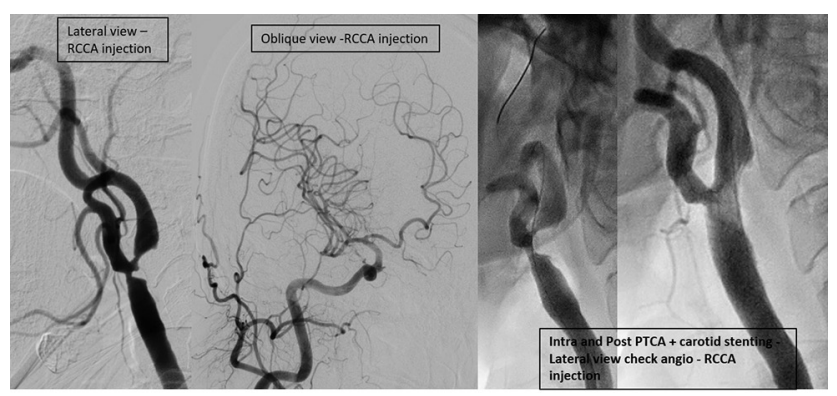

Fig. 8 Severe stenosis of carotid bifurcation and RICA origin are noted on the lateral views of the left common carotid artery (RCCA) injection, likely atherosclerotic. Cranial supply is normal as noted on the RCCA injection, oblique cranial view. Post percutaneous transluminal coronary angioplasty (PTCA) and stenting. Good flow distally. No haze/dissection/flap, as noted on the lateral views of the intraand postprocedural check RCCA angiograms.

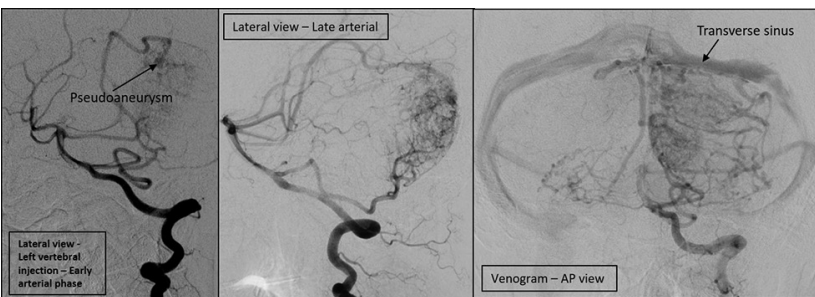

Fig. 9 There is evidence of an arteriovenous malformation (SM score 3 ) with diffuse nidus in the left cerebellar. Feeders from hemispheric branches of left superior cerebellar artery, posterior inferior cerebellar artery (hemispheric and vermian branches). Intranidal pseudoaneurysm (long arrow) noted on the posterosuperior aspect of the nidus. Venous drainage is through multiple cerebellar veins and superior vermian vein into the straight sinus and transverse sinus (short arrow).

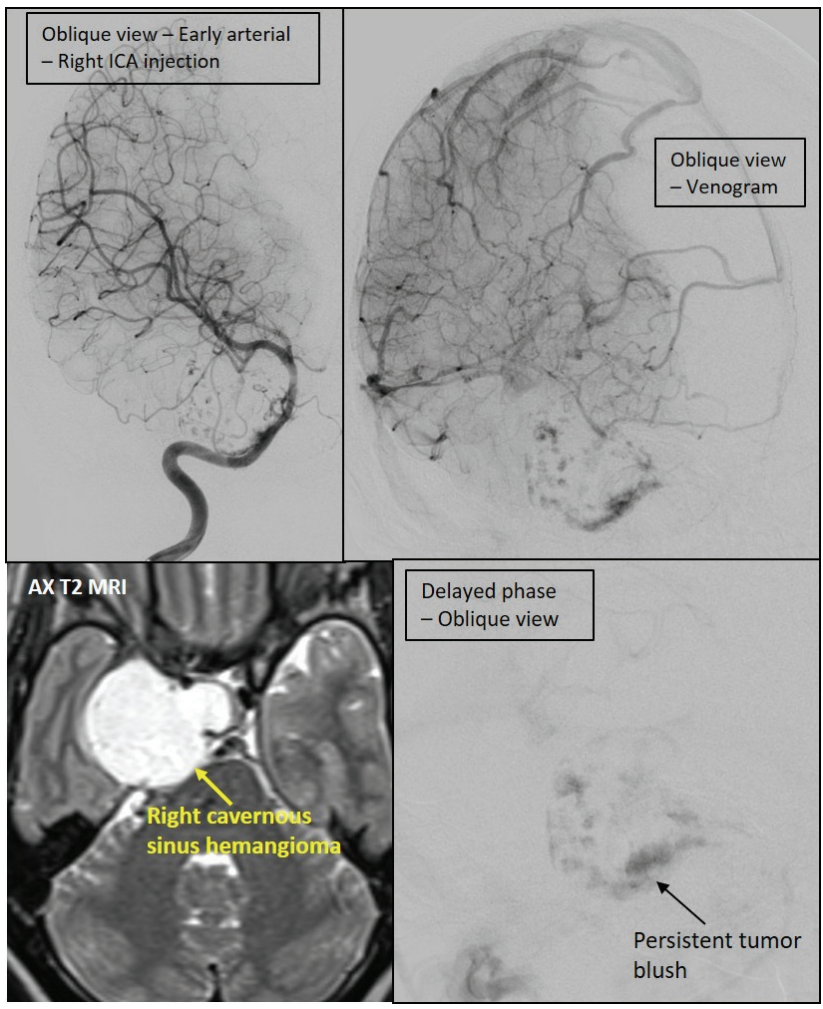

Fig. 10 Axial T2-weighted MRI showing a right parasellar homogenously bright lesion with mass effect, a known case of right cavernous sinus hemangioma (yellow arrow). Tumor blush (black arrow) seen in the right parasellar region with indistinct en passage feeders arising from dural branches of cavernous RICA (predominant feeder), distal right internal maxillary branches, and right middle meningeal artery. Mass effect on the adjacent RICA-widening of $\mathrm{C}$ loop with mildluminal narrowing. Patchy tumor blush and corkscrew pattern are seen appearing in the early arterial phase, progressively increasing and persisting into the delayed venous phase. Hypoplastic $A 1$ segment of the right ACA. ICA, internal carotid artery. 

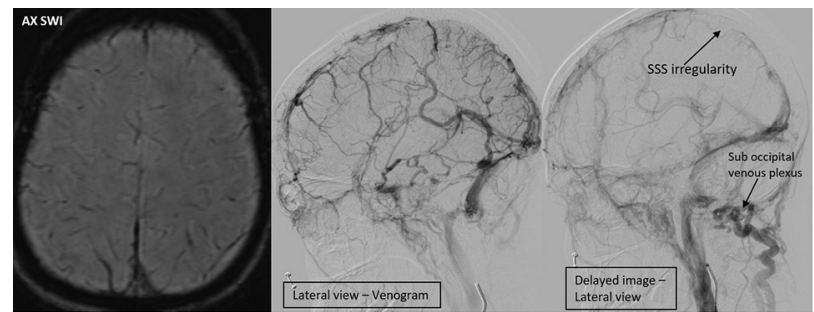

Fig. 11 Patient with chronic headache and seizures for $>10$ years. There is irregularity of the superior sagittal sinus (arrow), straight sinus, and bilateral transverse sinuses with venous rerouting through multiple diploic-meningeal veins, cavernous sinus to the pterygoid venous plexus and orbital veins, and also through the posterior fossa veins into the suboccipital venous plexus (downward pointing arrow) as noted on the lateral view of digital subtraction venograms. No evidence of dural AVF. Thus, a diagnosis of chronic dural venous sinus thrombosis was made.

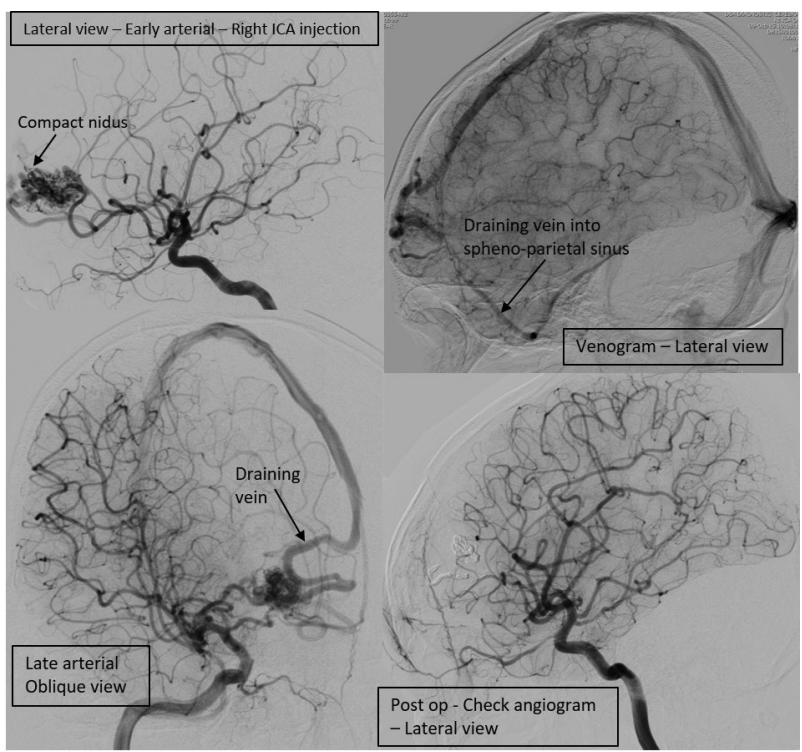

Fig. 12 There is evidence of compact nidus arteriovenous malformation (arrow) (SM score I) in the right frontal region fed by hypertrophied frontal branches of right ACA and MCA. Drainage is via a single cortical vein (arrow) into the anterior third of superior sagittal sinus and also via cortical vein into sphenoparietal sinus (arrow) and then into right transverse-sigmoid junction. Postoperative check angiogram shows no evidence of residual AVM/vessel spasm with clip artifact at the region of the nidus. ICA, internal carotid artery.

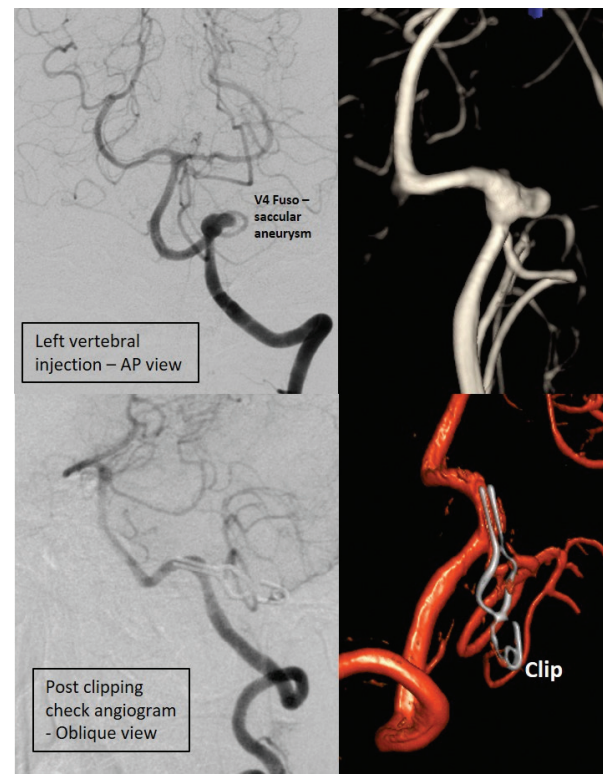

Fig. 13 There is evidence of a fusosaccular aneurysm arising from the V4 segment of left VA just distal to the origin of PICA. Saccular component is directed superolaterally. Post clipping check angiogram revealed a small residual proximal component of the aneurysm (superior to the clip). AP, anteroposterior.

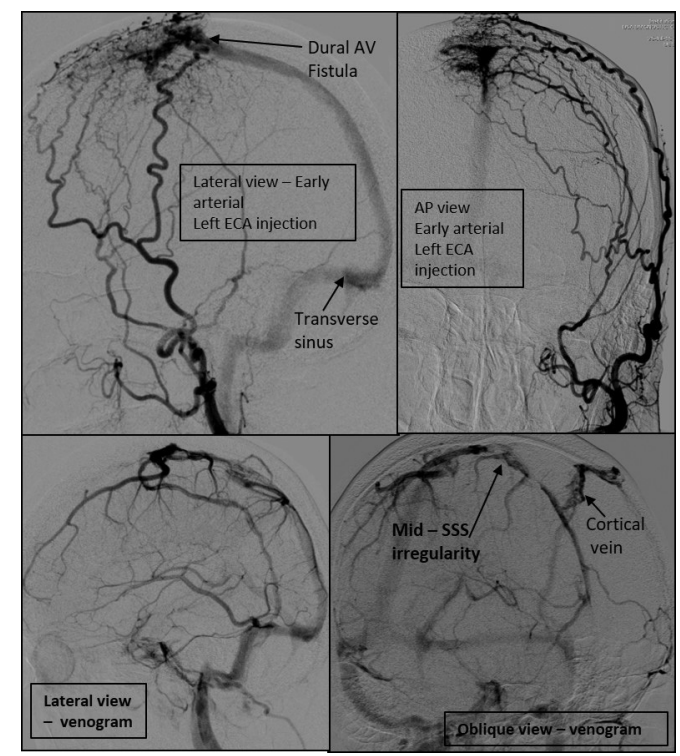

Fig. 14 There is evidence of a dural vascular malformation (horizontal arrow) with fistulous component at the mid-third of the superior sagittal sinus. Feeders to the malformation seen arising from en passage feeders from left middle meningeal artery and transosseous feeders from left superficial temporal artery and occipital artery. A short segment of mid-third of SSS appears irregular suggestive of thrombosed segment and partial recanalization. Drainage is seen into a cortical vein in the frontoparietal region and the SSS with predominant antegrade flow into the left transverse-sigmoid junction. AV, arteriovenous; ECA, external carotid artery. 


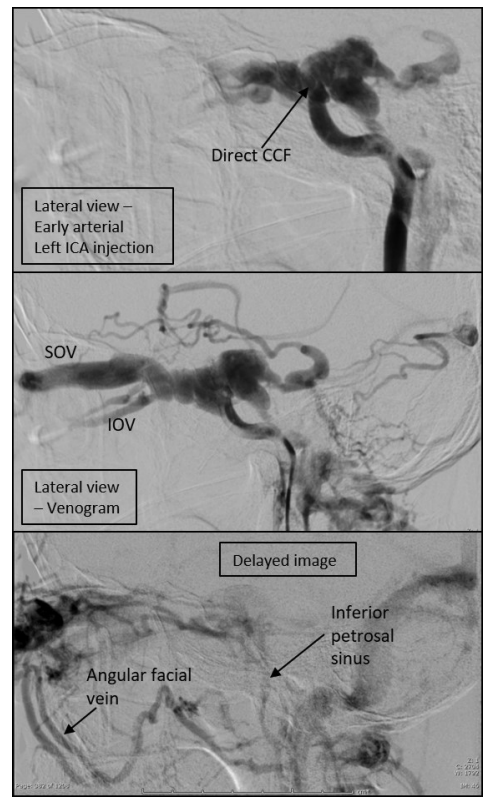

Fig. 15 There is evidence of high-flow direct caroticocavernous fistula (CCF, top arrow) with rent possibly along the posterior genu of the cavernous segment of internal carotid artery (ICA). Drainage is noted anteriorly through the dilated and tortuous superior (SOV) and inferior ophthalmic veins (IOV), then into facial vein (bottom left arrow), posteriorly into the inferior petrosal vein (bottom right arrow) and then into the internal jugular vein. Venous reflux is noted into the cortical veins into deep venous system separately into transverse sinus. Opacification of intracranial arteries is poor.

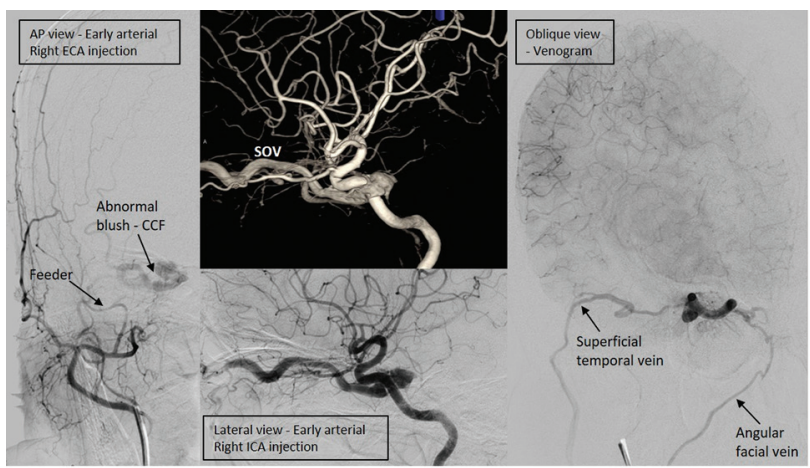

Fig. 16 There is evidence of a slow-flow right-sided indirect caroticocavernous fistula (CCF) (type D2) on left side being fed by dural branches arising from cavernous segment right internal carotid artery (ICA), distal/dural branches of right internal maxillary artery and middle meningeal. Drainage is antegrade; predominantly anterior through the superior ophthalmic vein (SOV) into angular vein, into the facial and superficial temporal veins. AP, anteroposterior; ECA, external carotid artery.

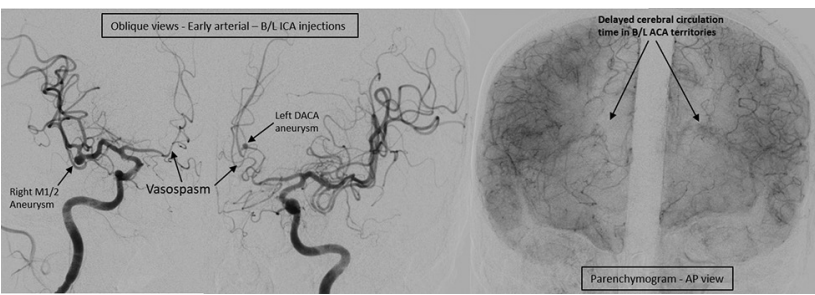

Fig. 17 There is evidence of saccular distal anterior cerebral artery (DACA) aneurysm arising at the bifurcation of anterior cerebral artery (ACA) into pericallosal and callosomarginal arteries, on the oblique view of the left internal carotid injection. There is marked spasm of bilateral ACAs with slow flow within and poor parenchymogram. The cerebral circulation time is significantly delayed. Another saccular aneurysm noted involving the M1/2 segment of the right MCA on the right internal carotid injection. AP, anteroposterior; ICA, internal carotid artery.

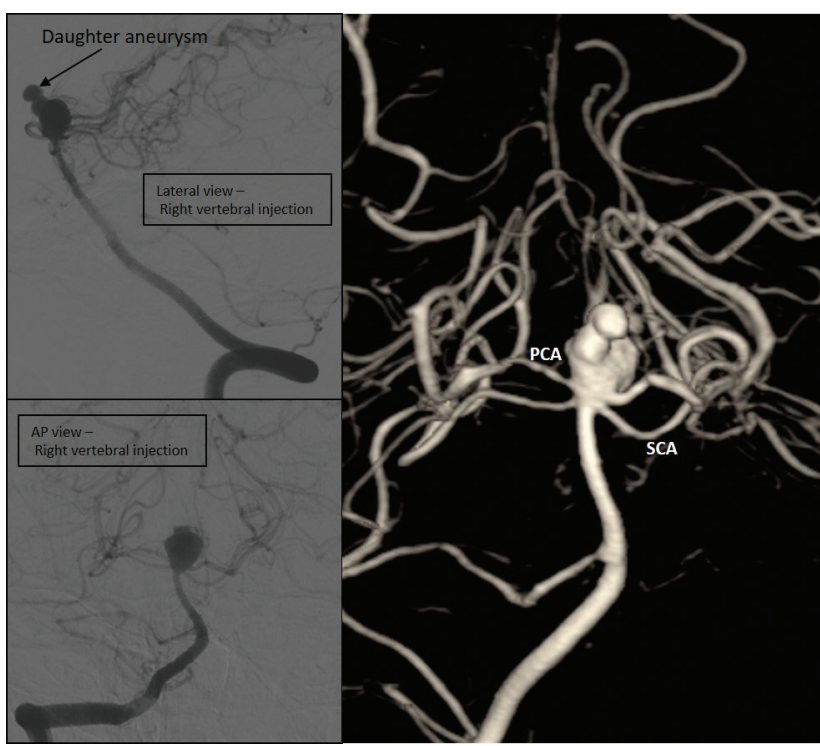

Fig. 18 Saccular basilar top aneurysm with eccentric outpouching (daughter aneurysm, arrow) at the apex. The superior cerebellar arteries (SCA) and posterior cerebral arteries (PCA) are noted separately from the sac. AP, anteroposterior.
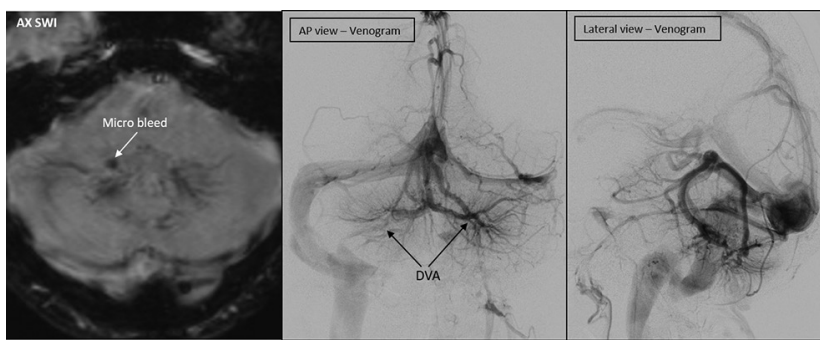

Fig. 19 Susceptibility-weighted MRI shows bilateral cerebellar parenchymal brush-like hypointensities (white arrow). On digital subtraction venogram images, bilateral large developmental venous anomalies (DVA, black arrows) noted with classic "Medusa head" appearance. AP, anteroposterior. 


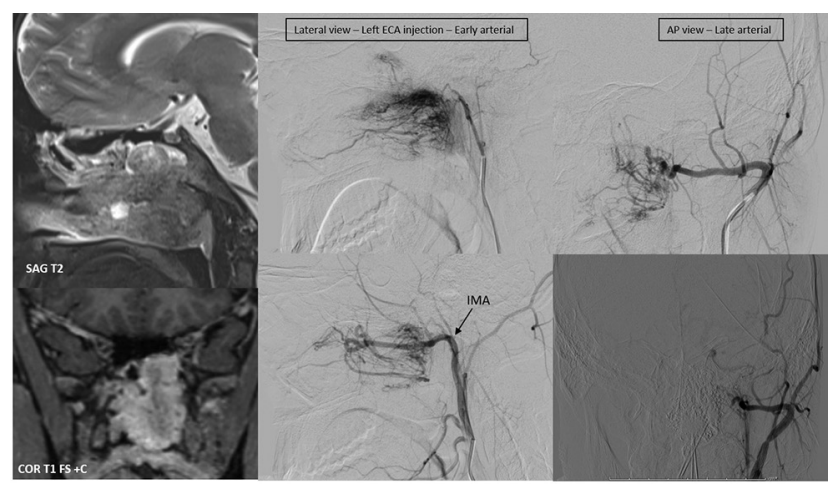

Fig. 20 MRI scans showing an intensely enhancing soft tissue lesion widening the sphenoethmoid recess. On DSA, there is evidence of a tumor blush with progressive filling and corkscrew-shaped intratumoral vessels, arterial feeders from the left external carotid branches (internal maxillary artery [IMA arrow] and ascending pharyngeal). Thus, a diagnosis of juvenile nasopharyngeal angiofibroma (JNA) was made. Postendovascular and percutaneous embolization; check angiogram shows no tumor blush. AP, anteroposterior; ECA, external carotid artery

\section{Conclusion}

Diagnostic neuroangiography is an essential investigation used in the evaluation of neurovascular diseases. Basic knowledge of angiographic technique and image interpretation are important for radiology residents, and our poster summarizes the essential information on this topic.

\section{Conflict of interest}

None.

\section{Informed Consent Statement}

Written informed consent was obtained from all patients for publication of this case report, including accompanying images.

\section{Acknowledgment}

The authors would like to acknowledge Dr. Vijay Chundi, $\mathrm{MD}(\mathrm{ABR})$, for his continued support and encouragement.

\section{References}

1 Thiex R, Norbash AM, Frerichs KU. The safety of dedicated-team catheter-based diagnostic cerebral angiography in the era of advanced noninvasive imaging. AJNR Am J Neuroradiol 2010;31(2):230-234

2 Basic neuroangiography review of technique and perioperative patient care; Sun Ho Ahn, MD. Semin Intervent Radiol 2013;30:225-233

3 Citron SJ, Wallace RC, Lewis CA, et al; Society of Interventional Radiology; American Society of Interventional and Therapeutic Neuroradiology; American Society of Neuroradiology. Quality improvement guidelines for adult diagnostic neuroangiography. Cooperative study between ASITN, ASNR, and SIR. J Vasc Interv Radiol 2003;14(9 Pt 2):S257-S262

4 Bouthillier A, van Loveren HR, Keller JT. Segments of the internal carotid artery: A new classification. Neurosurg 1996;38:425-432

5 Kaufman JA, Nesbit GM. Carotid and vertebral arteries. In: Kaufman JA, Lee MJ, eds. Vascular and Interventional Radiology: The Requisites. 2nd ed. Philadelphia, PA: Elsevier Saunders; 2014: 99-118 